\section{Virgilio Gallai \\ Paola Sarchielli}

Received: 15 October 2000

Accepted in revised form: 30 January 2001

V. Gallai $(\bowtie) \cdot$ P. Sarchielli

Neuroscience Department,

Policlinico Monteluce,

Via E. Dal Pozzo, I-06126 Perugia, Italy

e-mail: gallai@unipg.it

Tel.: +39-075-578-3568

Fax: +39-075-578-3583

\title{
Nitric oxide in primary headaches
}

\begin{abstract}
Nitric oxide (NO) has been proposed to be a key molecule in migraine. Experimental evidence suggests its intervention in vasodilatation and activation of the trigeminovascular system as well as its involvement in the supraspinal pathways implicated in head pain processing. Other findings suggest the implication of NO in coupling neuronal and vascular changes during spreading depression. A potential role for NO has also been proposed in the pathophysiological mechanisms underlying cluster headache and chronic tension-type headache. The most relevant evidence for an increased response to exogenous NO in all primary headaches emerges from the experimental model of nitroglycerin-induced headache. Moreover, the effectiveness of non-selective NO synthase inhibitor L- $N$-monomethylarginine (L-NMMA) further supports the involvement of NO in migraine and chronic tension-type headache. The endogenous increase in NO production has been shown only in studies carried out during spontaneous migraine attacks which demonstrated increased levels of nitrites and cGMP

cyclooxygenase (COX) due to NO. Additional evidence suggests the increased activity of L-arginine/NO pathway in the platelet model in migraine patients. This increase was also evident between attacks, more accentuated during attacks and expressed to a greater extent during late luteal phase in menstrual migraine. Higher NO production in platelet was also demonstrated in patients affected by chronic daily headache and they are accompanied by significant lower serotonin content and higher levels of intracellular calcium. Whether these changes may be expressed in the central nervous system is a matter of discussion. These data taken together suggest a crucial role played by $\mathrm{NO}$ in neurovascular headaches and chronic headaches. Further research concerning NO in all primary headaches will be aimed at verifying changes of reliable markers of NO metabolism and $\mathrm{NO}$ effects, to better understand the complex COX/NO synthase (NOS) interactions, to investigate the effectiveness of selective NOS inhibitors in discriminating neural versus vascular involvement of NO.
\end{abstract} in peripheral blood and internal jugular blood; the latter was followed by an increased production of algogenic and vasodilatatory prostanoids. These data suggest the potential activation of
Key words Primary headaches • Nitric oxide-induced headache $\cdot \mathrm{NO}$ markers $\cdot$ Cellular models $•$ Nitric oxide inhibitors 


\section{Introduction}

The origin and mechanisms of nociception in migraine and other primary headaches are not completely understood.

The large intracranial arteries are pain-sensitive structures: in man, mechanical dilatation during balloon angioplasty under local anesthesia gives rise to pain referred to areas where migraine pain is often experienced [1]. Therefore, both the quality and location of pain elicited from the large intracranial arteries are similar to the clinical features during migraine headache.

Friberg and colleagues [2], used a combination of transcranial Doppler (TCD) and single photon emission tomography (SPET) to measure middle cerebral artery (MCA) blood velocity and relative cerebral blood flow ( $\mathrm{rCBF}$ ) in the supply area of the MCA, during unilateral migraine headache. They reported a reduced flow velocity on the headache side only during attacks, without any $\mathrm{rCBF}$ variation. In a successive, extensive investigation, reduced velocity was definitely demonstrated on the headache side [3]. This evidence supports the association between unilateral pain in migraine and dilatation of the ipsilateral MCA, and suggests a vascular involvement during attacks.

In this scenario, the nitric oxide (NO) cascade is heavily involved, together with vasoactive peptides released from trigeminal endings after the activation of the trigemino-vascular system. $\mathrm{NO}$ is a powerful dilator and exerts strong effects in cerebrovascular regulation. NO mediates a great variety of physiological phenomena several of which, theoretically, are implicated in the pathophysiology of migraine (Table 1) $[4,5]$. This intra- and intercellular messenger has a very short half-life (5-15 seconds) and is a highly reactive free radical which is rapidly converted into stable metabolites, nitrites and nitrates. NO synthase (NOS) is the enzyme which catalyzes the synthesis of NO from guanidino nitrogen atoms of L-arginine. Two isoforms of NOS, the endothelial form (eNOS) and the neural form (nNOS), are constitutively expressed and calcium/calmodulin-dependent [5]. Moreover, glial cells contain an inducible form of NOS which is calcium/calmodulin-independent and is mainly stimulated by proinflammatory cytokines $[6,7]$.
The main NO effects, also found in cerebral blood vessels, are mediated by the activation of the soluble guanylate cyclase that causes an increase in the synthesis of cyclic guanosine 3',5'-monophosphate (cGMP). This is responsible for a reduction in the intracellular $\mathrm{Ca}^{2+}$ levels in the target muscle cells of the cerebral vessels inducing their relaxation [7].

Evidence from experimental animal models demonstrated that NO is an important transmitter in pain pathways [8]. Activation of these pathways, at least in the spinal cord, may be associated with upregulation of neural NOS and the generation of NO. On the other hand, NOS inhibitors decrease central sensitization in animal models of persistent pain [9].

In a recent report, NOS inhibition reduced FOS expression in the trigeminal complex of cats due to electrical stimulation of the superior sagittal sinus. These results support the involvement of NO in the activation of this complex which is implicated in primary headaches [10].

\section{The nitroglycerin-induced headache model}

According to Olesen et al. [11], NO may be considered a key molecule in the pathogenesis of migraine. They suggested that activation of the pathway involved in its synthesis represents a common mechanism in experimental headache models, such as nitroglycerin- and histamineinduced headaches which have been used for trigger attacks in migraineurs and cluster headache patients $[12,13]$.

In patients who have migraine with or without aura, nitroglycerin infusion induced an early headache (during and immediately after infusion) which was more severe than that recorded in healthy controls [14]. It also induced a late headache, several hours after infusion, that, in the majority of the cases, fulfilled International Headache Society (IHS) criteria for migraine without aura [14]. These data suggest that migraine with and without aura share the same NO-mediated common pathway of nociception and support experimental evidence showing that cortical spreading depression (CSD) determines NO liberation contributing to clarification of the pathophysiologic mechanisms of migraine with aura [15].

Table 1 Physiological effects mediated by NO of relevance to migraine headache

\author{
Endothelium: dependent vasodilatation \\ Neurogenic vasodilatation (NANC nerves) \\ Release of vasodilatatory peptides from cranial perivascular sensory neural endings \\ Modulation of excitatory aminoacid release in CNS pathways related to pain perception (primary and secondary hyperalgesia) \\ Coupling of vascular and neuronal changes underlying cortical spreading-depression
}

$N A N C$, nonadrenergic non-cholinergic; $C N S$, central nervous system 
A dilatation of mean cerebral arteries was observed in patients with migraine without aura in both the early and late phases following the infusion of nitroglycerin that can be reversed by sumatriptan. This $5-\mathrm{HT}_{1 \mathrm{~B} / 1 \mathrm{D}}$ agonist has been demonstrated to be effective in relieving headache induced by nitroglycerin [16].

In cluster headache patients, sublingual nitroglycerin is followed by a typical cluster headache attack only during the active period and is accompanied by an increase in calcitoningene related peptide (CGRP) levels indicative of the induction of the activation of the trigeminovascular system [17].

Recently, it has been demonstrated that nitroglycerin can induce headache also in chronic tension-type headache patients with an early component which is more severe compared with control individuals and a late component (which does not appear in controls), whose characteristics resembles those of spontaneous headache complained of by the patients. This finding suggests that NO is involved in the central sensitization in these patients which follows the peripheral sensitization due to the increased and sustained myofascial input in these patients [18].

Many substances capable of inducing migraine attacks, other than nitroglycerin, such as reserpine, $m$-chlorophenylpiperazine, fenfluramine and prostacyclin, were believed to act by stimulating endothelial NOS to produce NO [16].

The molecular and cellular events that mediate the most representative model of nitroglycerin-induced headache are not completely understood. NO released from nitroglycerin might be responsible for head pain, at least in the early phase. This radical was thought to act on both cerebrovascular endothelium and perivascular endings, also inducing the release of histamine from endothelium and mast cells. Additional mechanisms, not yet proven, may also be involved in the late stages of the nitroglycerin-induced headache, such as stimulation of vasoactive and algogenic prostaglandin synthesis, both in the endothelium and at perivascular sites [16].

A further mechanism which has been advocated to explain NO-induced headache especially in migraine is an alteration of the serotonin system. This has been demonstrated in animal models in which effects of the infusion of nitroglycerin was investigated before and after the injection of para-clorophenylalanine, a tryptophan hydroxylase inhibitor inducing a hyposerotoninergic status. This compound seems to facilitate the physiological and pathological NO-mediated response in meningeal and cerebral microvessels, supporting a potential role for serotonin depletion in the headache response to NO in migraineurs [19].

Central mechanisms also seem to be implicated in nitroglycerin-induced headache, since the drug activates a set of vegetative, neuroendocrine and nociceptive structures in the central nervous system [20].
The experimental model of the nitroglycerin-induced headache suggests the occurrence of hypersensitivity to exogenous NO (supplied by NO donors) in migraine. On the other hand, upregulation of the endogenous L-arginine/NO pathway and increased NOS expression, namely the constitutively expressed form, seem also to take place during spontaneous attacks [21]. This upregulation may involve peripheral sites such as endothelium and perivascular sensory endings, NOS-containing fibers from the nonadrenergic non-cholinergic (NANC) system supplying cerebral circulation, including not only the cerebral tissue surrounding cerebral vessels but also other spinal and supraspinal structures implicated in the transmission of head pain such as the trigeminocervical complex.

As far as eNOS and migraine occurrence are concerned, the upregulation of NO-generating pathways does not seem to be genetically determined in migraneurs [22]. Experimental data suggest that NO synthesis in endothelium is a consequence of an increased shear stress or may, alternatively, be activated and potentiated by the stimulation of specific receptors on endothelial cells by bradykinins, serotonin, acetylcholine, histamine, substance P (SP) and CGRP, all substances which are believed to be putative algogenic substances produced locally around vessels and mediating head pain [16].

NO has also been induced by contracting substances produced by endothelial cells, the most potent of which is endothelin 1 (ET-1) [23, 24].

The involvement of ET-1 in migraine has been demonstrated by our group. Higher levels of this vasoactive peptide were observed during the whole ictal phase, with a large increase in the first two hours [25].

This increase has been interpreted as a "physiological" response of endothelium in counteracting the vasodilatatory effect mediated by NO, prostacyclin and neuropeptides from the trigeminovascular system during migraine attacks or, alternatively, as a consequence of the prevalent activation of ET-1-mediated relaxation mechanisms.

\section{NO and trigeminovascular system}

In experimental models of peripheral neurogenic inflammation, NO seems to play a relevant role in edema formation, but not in vasodilatation, due to CGRP release [26, 27]. Moreover the relationship between endothelial NO production and release of the vasoactive peptides (CGRP, SP, neurokinin A) from the trigeminovascular system is only partly known.

Experimental animal models support the intervention of endothelial-derived NO not only in determining the basal dural vessel tone but also in increasing the electrically evoked flow mediated by CGRP released from trigeminal 
afferents [28]. The synergistic vasodilatatory effect exerted by NO and CGRP is, at least in part, due to the facilitation of CGRP release by NO. This synergistic effect is believed to contribute to sensitizing perivascular trigeminal afferent terminals and to the maintenance of the activation of the trigeminovascular system, considered the final pathway in migraine and cluster headache attacks.

The crucial role of NO from endothelial origin in mediating vessel basal tone and increasing electrically evoked flow in the dural vessels is further supported by efficacy of the non-selective NOS inhibitor L-NG-monomethyl L-arginine (which exerts its action also on endothelial NOS) in antagonizing vasodilatation. Conversely, the ineffectiveness of aminomethyltiazine (a NOS inhibitor selective for iNOS) and 7-nitroindazole (a NOS inhibitor selective for nNOS) excludes the involvement of eNOS and nNOS in electrically evoked flow increase of dural vessels [28].

Not all the available data concur in defining the relationship between the endothelial production of $\mathrm{NO}$ and the CGRP release from the trigeminovascular system. In some experimental models in fact, CGRP seems to mainly exert an endothelium-independent potent vasodilatatory effect which does not involve NO generation [29]. This effect seems to be mediated by the stimulation of cAMP which induces the hyperpolarization of endothelial cells followed by the activation of calcium-activated potassium channels $\left(\mathrm{BK}_{\mathrm{Ca}}\right)$ and ATP-sensitive potassium channels $\left(\mathrm{K}_{\mathrm{ATP}}\right)$ [30]. In other experimental models, however, activation of these channels appears to indirectly involve NO production by endothelium [31]. In contrast, further experimental data suggest that vasodilatation induced by CGRP, at least in the pial arteries of rats, is inhibited by endothelial injury, supporting the role of $\mathrm{NO}$ as the final mediator of the vasodilatory effects of this peptide from the trigeminovascular system [32, 33].

SP is a potent endothelium-dependent vasodilator particularly concentrated around the superior sagittal sinus [32-34]. Neurokinin A (NKA) is co-localized with SP in perivascular nerve fibers and cerebral vessels and has a similar, though less strong, vasodilatatory action [35].

Findings on the relationship between SP and NO are more consistent. Less data are available on NKA that seems to prevailingly exert its vasodilatatory effect by interacting with its own $\mathrm{NK}_{2}$ receptors [36].

The relaxant effect of substance $\mathrm{P}$ is known to involve the release of NO from endothelial cells. This effect has been demonstrated in human extracranial circulation but not in intracranial circulation [37]. Recent data from JansenOlesen and Edvinsson [38] suggest that the vasodilatatory response to this neuropeptide is lost after removal of the endothelium and after treatment with NOS inhibitors. The response abrogated by NOS inhibitors is restored after administration of L-arginine.

\section{NO and cerebral spreading depression}

CSD is a wave of initial neural excitation followed by substained depolarization of neural and glial cells that move at a rate of $2-5 \mathrm{~mm} / \mathrm{min}$ over the cerebral cortex [39]. This involves changes in local ionic environment, $N$-methyl Daspartate receptor activation and release of glutamate into the interstitium [40].

$\mathrm{NO}$ is recognized to be one of the major mediators potentiating glutamatergic transmission and might be involved in biochemical changes underlying CSD. This messenger has been suggested to play a central role in the experimental model of CSD intervening in the coupling of neural and vascular events which underlie the neural cortical dysfunction [41].

SD induces regular changes of cerebral blood flow (CBF) which consisted in four phases: a brief hypoperfusion before the direct current (DC) shift; a marked CBF rise during the DC shift; a subsequent protracted increase of CBF; and finally a prolonged CBF reduction (oligemia) [42].

The hypothesis that NO participates in the vascular changes associated with CSD has already been tested in several studies, but the interpretation of results is difficult due to the different experimental designs and the different species used. Colonna et al. [43] demonstrated the relevance of NO to CSD-related arterial dilatation in the rabbit. Goadsby et al. [41] were able to abolish CSD-associated hyperemia by systemic inhibition of NOS in cats using $N G$ nitro-L-arginine methyl ester.

One of the most relevant evidence for the involvement of the arginine-NO pathway in cerebrovascular regulation of CSD comes from a study of Fabricius et al. [44]. In their experimental model of CSD, NOS inhibition by intravenous and/or topical application of $N G$-nitro-L-arginine enhanced the brief initial hypoperfusion, but the CBF increase and the subsequent oligemia were unchanged. Conversely, L-arginine prevented the development of the prolonged oligemia after CSD but had no influence on the marked rise of CBF during CSD. These data suggest the modulation of CSD by NO synthase and the potential usefulness of arginine in restoring reduced $\mathrm{CBF}$, during spreading oligemia in humans, which may be of importance for the persistence of neurological deficits in migraine aura. This can be relevant especially in cases of prolonged aura, where cerebral ischemia can potentially lead to neuronal cell damage.

In the cat [45], glyceryl trinitrate infusion induced a biphasic NO release in the pial MCA perfusion territory, which is associated with a pial artery vasodilatation and increase in $\mathrm{rCBF}$.

In contrast, there are data which do not support the role played by NO in coupling CBF changes and metabolism during neural activation. In rats Zhang et al. [46] found that systemic NOS inhibition did not significantly influence CBF 
during CSD whereas Wolf et al. [47] showed that nitric oxide inhibition does not affect the perfusion response and the tissue $\mathrm{PO}_{2}$ during CSD, suggesting that other mechanisms may be involved in brain oxygenation changes in this condition.

It is important to emphasize that NO is not only a vasodilator but also a transmitter and that NMDA receptor activation, essential for the propagation of CSD and for transmembrane $\mathrm{Ca}^{2+}$ influx, is a proper stimulus for constitutively expressed NOS in endothelial cells and in the nervous system. The contribution of both endothelial and neural NOS in CSD should be further investigated by the use of selective NOS inhibitors.

\section{Markers of NO metabolism in peripheral blood of headache patients}

\section{NO end-products}

Several technical problems have delayed research on endogenous production of NO in patients affected by primary headaches. The major difficulty encountered in the study of NO is due to the inherent nature of this molecule, particularly its short half-life. Another important factor, which concurs with large variations in the results when measuring end-products of NO, is hemodilution. Dietary habits can also influence serum and plasma levels. Therefore a nitrate-restricted diet is recommended when studying endogenous variations in NO production. Due to these limitations, the search for end-products of NO metabolism in the peripheral blood has until now yielded inconsistent results.

The earliest findings of a slight increase in the peripheral levels of nitrites, consistent with a parallel increase in ET1 levels, came from a study by Nattero et al. [48] on migraine patients examined in interictical periods and during attacks. There is also evidence of an increase in nitrite levels in peripheral blood of migraine patients examined interictally [49].

Recently, an increase of nitrite levels during the active periods in the peripheral blood of patients with cluster headache has been demonstrated, supporting the involvement of NO in the pathophysiological mechanisms of this primary headache [50].

That nitrites can exactly express the biological effects of NO is also a matter of controversy. Sulphydrylic species, such as acetylcysteine, and the thiol groups of albumin can react with NO resulting in nitrosothiols which have a longer half-life than NO. These nitrosothiols preserve the same properties of endothelial-derived relaxing factors (EDRF) and account for the long-term effects of NO, as both a vasodilatory and anti-platelet agent [51]. There is no evidence of modifications in blood nitrosothiols levels in migraineurs assessed during attacks.

On the other hand, the fact that hemoglobin and other heme-containing proteins show high affinity to NO, contributing to scavenge this radical and prevent NO-induced activation of soluble guanylate cyclase, should be kept in mind [4]. The binding of NO to the heme group has not yet been investigated in migraine patients during or between attacks.

NO can be measured indirectly by the determination of its intracellular messenger, even in the peripheral blood. One study, carried out by Stepien and Chalimoniuk [52], took this into consideration and demonstrated increased peripheral blood levels of cGMP in migraine patients assessed during the attacks. The levels of this cyclic nucleotide correlated with the pain intensity and showed a dramatic decrease after sumatriptan administration.

It has been hypothesized that there is a close relationship between the activation of the L-arginine/NO pathway and production of vasoactive and algogenic prostaglandins during spontaneous migraine attacks, but this suggestion needs to be confirmed. The temporal variations of NO end-products and vasoactive prostaglandins as well as vasoactive neuropeptides from the trigeminal vascular system during attacks should also be investigated.

In this regard, our group recently determined nitrites with HPLC and $\mathrm{PGE}_{2}$ and 6 keto PGF1 $\alpha$, the stable product of $\mathrm{PGI}_{2}$ with RIA method in the internal jugular venous blood of 5 patients affected by migraine without aura assessed during attacks [53]. Nitrite and cGMP levels reached their highest values at the first hour, then they decreased progressively and returned, after the end of the attacks, to values similar to or below those detected at the time of catheter insertion. Similar variations were observed for the two vasoactive peptides CGRP and NKA from the trigeminal vascular system. $\mathrm{PGE}_{2}$ and 6 keto $\mathrm{PGF} 1 \alpha$ as well as cAMP levels also significantly increased at the first hour but reached a peak at the second hour and remained in the same range until the fourth and sixth hours.

For the first time, these findings support an early activation of the L-arginine/NO pathway which accompanies the release of vasoactive peptides from trigeminal endings and a late rise in the synthesis of prostanoids with algogenic and vasoactive properties which may contribute to maintain the headache phase during migraine attacks.

\section{Cellular models}

Cellular models of peripheral blood were proposed for investigating NO relevance in migraine, and more recently, in chronic daily headache $(\mathrm{CDH})$. 
Platelets are a useful model not only for studying serotoninergic metabolism but also NO metabolism in migraine patients [54]. When stimulated by collagen, platelets produce NO, using L-arginine as a substrate [55]. D'Andrea et al. showed increased levels of L-arginine in the platelets of migraine patients, particularly in those with aura assessed in interictal periods, along with a reduced responsiveness to collagen [56]. These results support the hypothesis of an increased activity of the L-arginine/NO pathway in this pathological condition.

Based on these observations, our group investigated variations in ictal and interictal platelet NO production by measuring the steichiometric conversion of oxyhemoglobin to methemoglobin due to NOS activity and platelet cGMP production in migraine patients with and without aura, as compared to those of a group of age-matched control individuals [57]. Furthermore, the relationship between these variations and changes in platelet responsiveness to collagen, both ictally and interictally, were evaluated in the same patients. We found a reduced responsiveness to collagen in migraine patients, which was more accentuated during attacks. This reduced collagen response was coupled to a significantly higher basal and collagen-stimulated production of NO and cGMP in the platelet cytosol. This increased production was further accentuated during migraine attacks. We hypothesised that the variations in the L-arginine/NO pathway found in the platelets of migraine patients could be one of the metabolic counterparts of the pathophysiological alterations in platelet function and metabolism observed in migraineurs. This could indirectly reflect a compensatory mechanism intervening in the downregulation of platelet responsiveness after repeated stimulation by activating factors.

Taking into account these findings, we also determined the rate of production of NO and cGMP in the cytosol of platelets stimulated by collagen in females with menstrual migraine, assessed interictally and ictally, in the follicular and luteal phases as well as at mid-cycle [58]. An increased platelet NO production was demonstrated at the luteal phase in menstruation-related migraine patients. We hypothesized that this increase could be expressed also at the central level and could be involved in conditioning the great susceptibility to migraine attacks during perimenstrual and menstrual periods in these patients.

Shimomura et al. [59] further supports the increased activation of the L-arginine/NO pathway in patients affected by migraine with and without aura. The authors showed high levels of nitrite, total nitrate and nitrite as well as cGMP levels in platelet cytosol during attacks. These levels decreased after treatment with oral propranolol.

Further research from our group concerned the variations in L-arginine/NO pathway activity and the platelet cGMP levels in patients affected by chronic daily headache (CDH) [60]. A reduction in platelet aggregation response was found for every collagen concentration. This was coupled with increased NO and cGMP production and was greater than that previously observed in patients with episodic migraine. The activation of platelet L-arginine/NO pathway was accompanied by a reduced platelet content of serotonin and a concomitant increase in cytosolic $\mathrm{Ca}^{2+}$ levels, particularly in $\mathrm{CDH}$ patients with analgesic abuse.

We suggested that the upregulation of the L-arginine NO pathway is not limited to migraine being implicated, even to a greater extent, in $\mathrm{CDH}$. In this regard, it is necessary to mention that all $\mathrm{CDH}$ patients evolved from a previous history of migraine and that drug abuse contributed to the L-arginine/NO pathway overactivity maintaining pain chronicity.

Our findings therefore support the occurrence of NOcGMP mediated events in $\mathrm{CDH}$, perhaps as a compensatory mechanism for the cytosolic $\mathrm{Ca}^{2+}$ increase observed in platelets. This compensatory mechanism, however, seems not to be so efficient in contrasting high platelet $\mathrm{Ca}^{2+}$ concentration and could be responsible for serotonin depletion. This has recently been shown in platelets of patients affected by $\mathrm{CDH}$ associated with analgesic abuse [61]. Whether these events also occur in central pathways involved in pain transmission and nociception remains to be established.

Martelletti et al. proposed mononuclear cells as a cellular model for revealing involvement of the nitric oxide pathway in migraine pathophysiology [62]. They found higher nitrite levels in the culture medium of these cells in the interictal period and conversely a decreased nitrite level during NO donor-induced migraine attacks.

\section{Therapeutic aspects}

\section{NO and triptans}

Recent research aimed at elucidating the role of triptans in modulating NO during migraine attacks. The effect of sumatriptan in inhibiting nitroglycerin-induced headaches in humans is well known as well as reversing cGMP increase during migraine attacks $[52,63]$. There is also evidence of a reduction in the cortical regional blood flow and NO concentration and a concomitant increase in superoxide levels after sumatriptan administration in rats infused with nitroglycerin [64]. These results suggest that sumatriptan could modulate cell redox state and NO scavenging can be hypothesized. It is difficult though to believe that this effect will mediate the anti-migraine effect of the drug primarily at the neural level, taking into account the difficulty of the drug in crossing the blood-brain barrier.

On the other hand, there is evidence that the $5 \mathrm{HT}_{1 \mathrm{~B} / 1 \mathrm{D}}$ agonists sumatriptan and zolmitriptan eliminated the NMDA receptor-induced enhancement of cGMP in cortical slices in vitro. This mechanism needs to be further investigated in vivo, particularly in the context of the effectiveness of these drugs in relieving migraine attacks [65]. 
Nitric oxide synthase inhibition

The involvement of NO in initiating and maintaining migraine attacks has been furthermore demonstrated by the effectiveness of a NOS inhibitor L-NG-monomethyl L-arginine (L-NMMA) in treating migraine attacks. A recent double-blind controlled study $[66,67]$ showed that L-NMMA infusion induced a significant improvement of migraine pain and accompanying symptoms such as phonophobia and photophobia within 30 minutes. This positive effect was observed in $66 \%$ of the patients treated with the drug versus a placebo effect of $14 \%$.

Regarding the effectiveness of L-NMMA in migraine, it should be noted that L-NMMA is not specific for the neural or endothelial form of NOS. It cannot therefore be excluded that both neural and endothelial mechanisms could be influenced in the analgesic effect of NOS inhibition in migraine. The effect of the drug in these patients seems not to be mediated by vasocontriction of cerebral vessels because the flow velocities in the middle cerebral arteries measured by transcranial Doppler were not affected [66].

NOS inhibition was also proposed as a treatment for chronic tension-type headache based on the assumption that the central sensitization due to prolonged nociceptive input from pericranial myofascial tissues could play a role in the pathophysiology of this disorder. Taking this into account, Ashina et al. [68] undertook a double-blind crossover trial in 16 patients with chronic tension-type headache using LNMMA or placebo. They demonstrated that $120 \mathrm{~min}$ after administration mean pain scores were significantly decreased in patients treated with drug compared with placebo. Pain intensity on the verbal rating scale was also lowered by treatment with L-NMMA. In addition, muscle hardness was significantly reduced at 60 and $120 \mathrm{~min}$, but this did not occur in the group treated with placebo [69]. Increased muscle hardness in patients with chronic tension-type headaches has been attributed to a reflect sensitization of the secondorder neurons due to the prolonged nociceptive input from myofascial tissues. According to the authors the decrease in muscle hardness after L-NMMA administration could be due to a reduced central sensitization in the spinal horn. The antinociception action of L-NMMA seems not to be related to the vasoactive effect of this NOS inhibitor.

\section{SB-22043}

The new benzopiran SB-22043 exhibits a high affinity for a selective, but not yet characterized, binding site in the human brain. SB-22043 inhibits NO release and cerebral vasodilatation following CSD as well as carotid vasodilatation induced by trigeminal nerve stimulation in the cat, without a direct effect on the middle meningeal artery. Therefore this compound exerts its potential anti-migraine action by inhibiting cortical excitability and trigeminal pathway activation via an NO-producing pathway [70, 71].

\section{Conclusions}

Experimental data suggest the involvement of NO in the pathophysiological cascade of primary headaches, although the mechanisms of this interaction, in both inducing and maintaining head pain, are only speculative. Following the pathophysiological events taking place in head pain (Fig. 1) it can, at least, be argued, that NOS acts as a protagonist at different levels of the cascade.

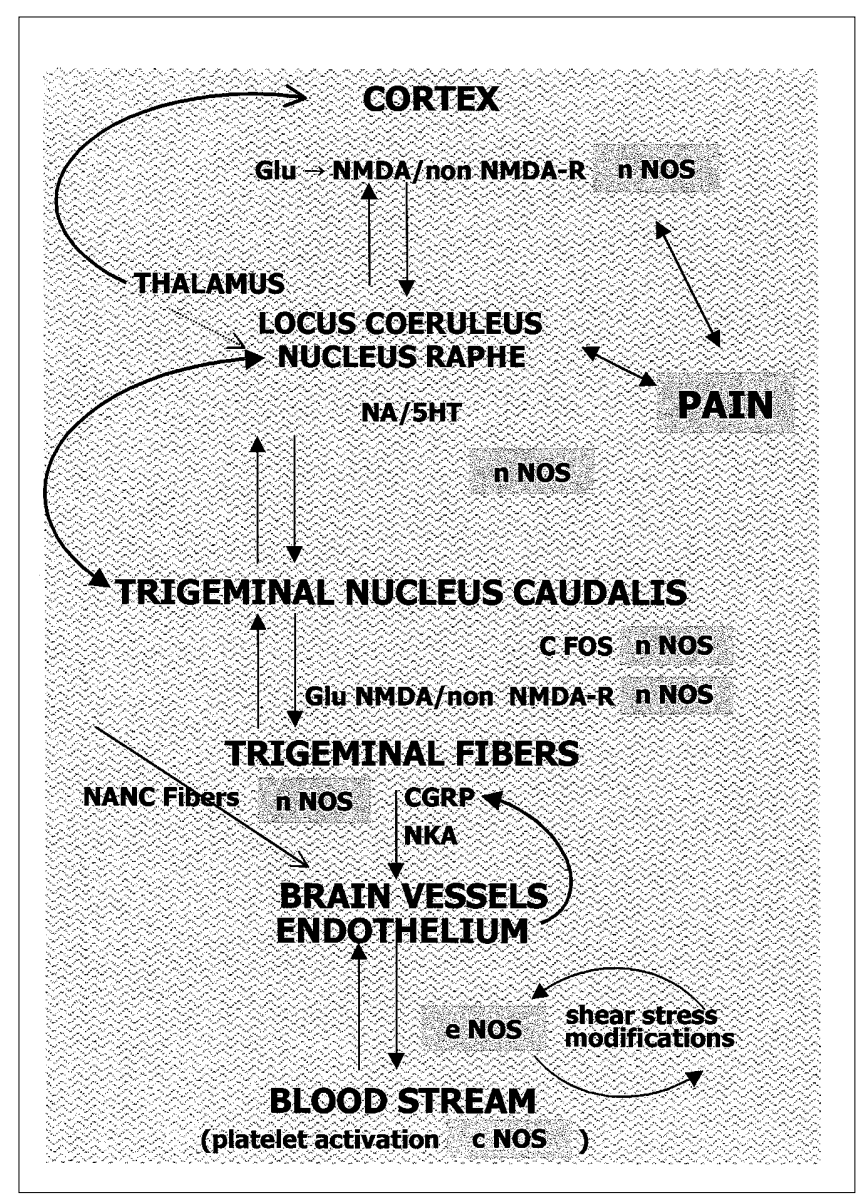

Fig. 1 Involvement of NOS in the pathophysiological cascade of head pain. CGRP, calcitonin gene related peptide; $c$ NOS, constitutively expressed NOS; e NOS, endothelial NOS; Glu, glutamate; $N A$, noradrenaline; $N A N C$, nonadrenergic, non-cholinergic; $N K A$, neurokinin A; NMDA-R, $N$-methyl D-aspartate receptor; $N O S$, nitric oxide synthase; $n N O S$, neural NOS; $5 H T$, 5-hydroxytryptamine 
In order to identify the actual role of NO in primary headaches it is crucial to have reliable markers. For this purpose, the most promising substances, which can be measured in different biological fluids, are nitrites/nitrates, cGMP and nitrosothiols. The NO-heme complex can also be investigated by means of electron paramagnetic resonance (EPR).

Another primary need is to further develop cellular models, since they permit (i) to study at the same time NOS pathway and related neurotransmitters and mediators, and (ii) to extrapolate pathophysiological data which could reflect analogous phenomena taking place in CNS. In this context, special effort should be spent for a better understanding of COX/NOS interactions.

Great attention has been paid up until now to constitutively expressed NOS, both endothelial and neural; perhaps, also inducible forms could play some role. To this purpose, an exciting perspective may be offered by experimental models which can allow us to investigate the role played by glial cells, which are a natural reservoir of many NOSinducing substances.

Finally, the use of selective NOS inhibitors can be proposed for discriminating neural versus vascular involvement of NO in primary headaches; this would have relevant clinical implications, not only in the field of headache.

Considering the clinical and experimental data herein presented, nitric oxide certainly represents a piece of the complex mosaic of the pathophysiological mechanisms intervening in primary headaches. Although its role has been partially elucidated, research is continually moving towards a better understanding of the relationship between this intracellular and intercellular messenger and the other pieces trying to reach a unified view.

\section{References}

1. Nichols FT III, Mawad M, Mohr JB, Stein B, Hilal S, Michelsen J (1990) Focal headache during balloon inflation in the internal carotid and middle cerebral arteries. Stroke 21:555-559

2. Friberg L, Olesen J, Iversen HK, Sperling B (1991) Migraine pain associated with middle cerebral artery dilatation: reversal by sumatriptan Lancet 338:13-17

3. Thomsen LL, Iversen HK, Olesen J (1993) Cerebral blood flow velocities are reduced during attacks of unilateral migraine without aura. Cephalalgia 15:109-116

4. Moncada S, Palmer RMJ, Higgs EA (1991) Nitric oxide: physiology, pathophysiology and pharmacology. Pharmacol Rev 43:127-136

5. Thomsen LL (1997) Investigation into the role of nitric oxide and the large intracranial arteries in migraine headache. Cephalalgia 17:873-895

6. Mayer B (1994) Regulation of nitric oxide synthase and soluble guanylyl cyclase. Cell Biochem Funct 12:167-177

7. Kiechle FL, Malinski T (1993) Nitric oxide biochemistry, pathophysiology and detection. Am J Clin Pathol 100:567-573

8. Luo ZD, Cizkova D (2000) The role of nitric oxide in nociception. Curr Rev Pain 4(6):459-66
9. Meller ST, Gebhart GF (1993) Nitric oxide (NO) and nociceptive processing in the spinal cord. Pain 52:127-136

10. Hoskin KL, Bulmer DC, Goadsby PJ (1999) Fos expression in the trigeminal complex of the cat after stimulation of the superior sagittal sinus is reduced by L-NAME. Neurosci Lett 266(3):173-176

11. Olesen J, Thomsen LL, Iversen HK (1994) Nitric oxide is a key molecule in migraine and other vascular headaches. Trends Pharmacol Sci 15:149-153

12. Olesen J, Thomsen LL, Lassen LH, Olesen IJ (1995) The nitric oxide hypothesis of migraine and other vascular headaches. Cephalalgia 15:99-100

13. Lassen LH, Thomsen LL, Olesen J (1995) Histamine induces migraine via the H1-receptor. Support for the NOhypothesis of migraine. Neuroreport 6:1475-1479

14. Christiansen I, Thomsen LL, Daugaard D, Ulrich V, Olesen J (1999) Glyceryl trinitrate induces attacks of migraine without aura in sufferers of migraine with aura. Cephalalgia 19(87):660-667

15. Urenjak J, Obrenovitch TP (2000) Pharmacological investigation into the involvement of nitric oxide in $\mathrm{K}^{+}$induced cortical spreading depression. Naunyn Schmiedebergs Arch Pharmacol 362(2):137-44
16. Thomsen LL, Olesen J (1998) Nitric oxide theory of migraine. Neuroscience 5:28-33

17. Fanciullacci M, Alessandri M, Sicuteri R, Marabini S (1997) Responsiveness of the trigeminal system to nitroglycerine in cluster headache. Brain 120:283-288

18. Ashina M, Bendtsen L, Jensen R, Olesen J (2000) Nitric oxide-induced headache in patients with chronic tension-type headache. Brain 123:1830-1837

19. Srikiatkhachorn A, Anuntasethakul T, Maneesri S, Phansuwan-Pujito P, Patumraj S, Kasantikul V (2000) Hyposerotoninergic-induced nitric oxide supersensitivity in the cerebral microcirculation. Headache 40:267-275

20. Tassorelli C, Joseph SA. Buzzi MG, Nappi G (1999) The effects on the central nervous system of nitroglycerin putative mechanisms and mediators. Prog Neurobiol 57(6):607-624

21. Olesen J, Iversen HK, Thomsen LL (1993) Nitric oxide supersensitivity. A possible molecular mechanism of migraine pain. Neuroreport 15:94-100

22. Griffith LR, Nyholt DR, Curtain RP, Goadsby PJ, Brimage PJ (1997) Migraine association and linkage studies of an endothelial nitric oxide synthase (NOS3) gene polymorphism. Neurology 49:614-617 
23. Inoue $A$, Yanagisawa $M$, Rimura $S$ et al (1989) The human endothelin family: three structurally and pharmacologically distinct isopeptides predicted by three different genes. Proc Natl Acad Sci USA 86:2863-2867

24. Haynes WG, Webb DJ (1993) The endothelin family of peptides: local hormones with diverse roles in health and disease? Clin Sci 84:485-500

25. Gallai V, Sarchielli P, Firenze C, Trequattrini A, Paciaroni M, Usai F, Palumbo R (1994) Endothelin 1 in migraine and tension-type headache. Acta Neurol Scand 89:47-55

26. Kajekar R, Moore PK, Brain SD (1995) Essential role of nitric oxide in neurogenic inflammation in rat cutaneous microcirculation. Evidence for an endothelium-independent mechanism. Circ Res 76:441-447

27. Edvinsson L, Mulder H, Goadsby PJ, Uddman R (1998) Calcitonin generelated peptide and nitric oxide in the trigeminal ganglion: cerebral vasodilatation from trigeminal nerve stimulation involves mainly calcitonin generelated peptide. J Auton Nerv Syst 70(1-2):15-22

28. Messlinger K, Suzuki A, Pawlak M, Zehnter A, Schmidt RF (2000) Involvement of nitric oxide in the modulation of dural arterial blood flow in the rat. Br J Pharmacol 129(7):1397-1404

29. Baskaya MK, Duzuki Y, Anzai M, Seki Y, Saito K, Takayasu M et al (1995) Effect of adrenomedullin, calcitonin gene related peptide, and amylin on cerebral circulation in dogs. J Cereb Blood Flow Metab 15:827-834

30. Holland M, Langton PD, Standeh NB, Boyle JP (1996) Effects of BK $\mathrm{Ca}$ channel activator, NS1619, on rat cerebral artery smooth muscle. Br J Pharmacol 117:119-129

31. Hoang LM, Mathers DA (1998) Internally applied endotoxin and the activation of BK channels in cerebral artery smooth muscle via a nitric oxidelike pathway. Br J Pharmacol 123:5-12

32. Rosemblun WI, Shimizy T, Nelson GH (1993) Endothelium-dependent effect of substance $\mathrm{P}$ and calcitonin generelated peptide on mouse pial arterioles. Stroke 24:1043-1048
33. Zawadzki JV, Furchgott RF, Cherry P (1981) The obligatory role of endothelial cells in the relaxation of arterial smooth muscle by substance P Fed Proc 40:689

34. Zawadzki JV, Furchgott RF, Cherry P (1981) The obligatory role of endothelial cells in the relaxation of arterial smooth muscle by substance P. Fed Proc 40:689

35. Keller JT, Marfurt CF (1989) Peptidergic and serotoninergic innervation of the rat dura mater. J Comp Neurol 290:310-321

36. Edvinsson L, Brodin E, Jansen I, Uddman R (1988) Neurokinin A in cerebral vessels: characterization, localization and effects in vitro. Regul Peptides 20:181-197

37. Furchgott RF (1984) The requirement for endothelial cells in the relaxation of arteries by acetylcholine and some other vasodilators. Trends Pharmacol Sci 2:173-176

38. Jansen-Olsen I, Edvinsson L (2000) Cephalic neurovascular transmitters and receptors. In: Olesen J, TfeltHansen P, Welch KMA (eds) The headaches, 2nd edn. Lippincott Williams \& Wilkins, Philadelphia, pp

39. Leao AAP (1944) Spreading depression of activity in the cerebral cortex. J Neurophysiol 7:359-390

40. Fabricius M, Jensen LH, Lauritzen M (1993) Microdialysis of interstitial amino acids during spreading depression and anoxic depolarisation in rat neocortex. Brain Res 612:61-69

41. Goadsby PJ, Kaube H, Hoskin KL (1992) Nitric oxide synthesis couples cerebral blood flow and metabolism. Brain Res 595:167-170

42. Duckrow RB (1991) Regional cerebral blood flow during spreading cortical depression in conscious rats. J Cereb Blood Flow Metab 11:150-154

43. Colonna DM, Meng W, Deal DD, Busija DW (1994) Nitric oxide promotes arteriolar dilation during cortical spreading depression in rabbits. Stroke 25:2463-2470

44. Fabricius M, Akgoren N, Lauritzen M (1995) Arginine-nitric oxide pathway and cerebral regulation in cortical spreading depression. Am J Physiol 269:H23-H29
45. Read SJ, Smith AJ, Hunter AJ, Parsons AA (1997) Enhanced nitric oxide release during cortical spreading depression following infusion of glyceryl trinitrate in the anaesthetized cat. Cephalalgia 17:159-165

46. Zhang ZG, Chop M, Maynard KI, Moskowitz A (1994) Cerebral blood flow changes during cortical spreading depression are not altered by inhibition of nitric oxide synthesis. J Cereb Blood Flow Metab 14:939-943

47. Wolf T, Lindauer U, Obrig H, Dreier J, Back T, Villringer A, Dirnag U (1996) Systemic nitric oxide synthase inhibition does not affect brain oxygenation during cortical spreading depression in rats: a noninvasive near-infrared spectroscopy and laser-Doppler flowmetry study. J Cereb Blood Flow Metab 16:100-1107

48. Nattero G, Mencossi G, Ianconis T, Paradisi L (1996) Nitric oxide, endothelin 1 and transcranial Doppler in migraine. Findings in interictal conditions and during migraine attacks. Headache 36(5):307-311

49. D'Amico D, Ferraris A, Catania A, Carlin A, Leone M, Attanasio A, Bussone G (1998) Increased basal nitric oxide plasma concentration in migraine. EOS J Immunol Immunopathol 18:109-111

50. D'Amico D, Leone M, Ferraris A, Catania A, Carlin A, Grazzi L, Bussone G (1999) Role of nitric oxide in cluster headache. Ital J Neurol Sci 20(7):S25-S27

51. Keaney JF Jr, Simon DI, Stamler JS, Jakari O, Scharfstein J, Vita JA, Lo Scalzo J (1993) NO forms an adduct with serum albumin that has endothelium-derived relaxing factor-like properties. J Clin Invest 91(4):1582-1589

52. Stepien A, Chalimoniuk M (1998) Levels of nitric oxide-dependent cGMP in patients with migraine. Cephalalgia 18(9):631-634

53. Sarchielli P, Alberti A, Codini M, Floridi A, Gallai V (2001) Nitric oxide metabolites, prostaglandins and trigeminal vasoactive peptides in internal jugular vein blood during spontaneous migraine attacks. Cephalalgia (in press) 
54. Sarchielli P, Gallai V (1998) Platelet Larginine/nitric oxide pathway in migraine. EOS J Immunol Immunopathol 18:78-85

55. Radomski MW, Palmer RMJ, Moncada S (1990) L-arginine/nitric oxide pathway present in human platelets regulates aggregation. Proc Natl Acad Sci USA 87:5193-5197

56. D'Andrea G, Cananzi AR, Perini F, Alecci M, Zamberlan F, Hasselmark L, Welch KMA (1994) Decreased collagen-induced platelet aggregation and increased platelet arginine levels in migraine: a possible link with the NO pathway. Cephalalgia 14:352-356

57. Gallai V, Floridi A, Mazzotta G, Codini M, Tognoloni M, Ambrosini P, Sartori M, Russo S, Feleppa M, Sarchielli P (1996) L-arginine/nitric oxide pathway activation in platelets of migraine with and without aura patients. Acta Neurol Scand 94:151-160

58. Sarchielli P, Tognoloni M, Russo S, Feleppa M, Malà M, Sartori M, Gallai V (1996) Variations in the platelet nitric oxide synthase pathway during the ovarian cycle in females affected by menstrual migraine. Cephalalgia 16:450-458

59. Shimomura T, Murakami F, Kotani K, Ikawa S, Kono S (1999) Platelet nitric oxide metabolites in migraine. Cephalalgia 19:218-222

60. Sarchielli P, Alberti A, Tognoloni M, Russo S, Firenze C, Gallai V (1999) Nitric oxide pathway, $\mathrm{Ca}^{+}$and serotonin content in platelets from patients suffering from chronic daily headache. Cephalalgia 19:800-806
61. Srikiatkhachon A, Maneesri S, Govitrapong P, Kasantikul V (1998) Derangement of serotonin system in migrainous patients with analgesic abuse headache: clues from platelets. Headache 38:43-49

62. Martelletti P, D'Alò S, Stirparo G, Cifone MG, Giacovazzo M (1998) Modulation of nitric oxide synthase by nitric oxide donor compounds in migraine. Int J Clin Lab Res 28:135-139

63. Iversen HK, Olesen J (1996) Headache induced by nitric oxide donor (nitroglycerin) responds to sumatriptan. A human model for the development of migraine drugs. Cephalalgia 16(6):412-418

64. Read SJ, Manning P, McNeil CJ, Hunter AJ, Parsons AA (1999) Effects of sumatriptan on nitric oxide and superoxide balance during glyceryl trinitrate infusion in the rat. Implication for antimigraine mechanisms. Brain Res 847(1):1-8

65. Stepien A, Chalimoniuk M, Strosznajder J (1999) Serotonion $5 \mathrm{HT}_{1 \mathrm{~B} / 1 \mathrm{D}}$ receptor agonists abolish NMDA receptor-evoked enhancement of nitric oxide synthase activity and cGMP concentration in brain cortex slices. Cephalalgia 19:859-865

66. Lassen LH, Ashina M, Christiansen I, Ulrich V, Olesen J (1997) Nitric oxide synthase inhibition in migraine. Lancet 349:401-402
67. Lassen LH, Ashina M, Christiansen I, Ulrich V, Grover R, Donaldson J, Olesen J (1998) Nitric oxide synthase inhibition: a new principle in the treatment of migraine attacks. Cephalalgia 18(1):27-32

68. Ashina M, Bendtsen L, Jensen R, Lassen LH, Sakai F, Olesen J (1999) Effect of inhibition of nitric oxide synthase on chronic tension-type headache: a randomised crossover trial. Lancet 353:287-289

69. Ashina M, Bendtsen L, Jensen R, Lassen LH, Sakai F, Olesen J (1999) Possible mechanisms of action of nitric oxide synthase inhibitors in chronic tension-type headache. Brain 122:1629-1635

70. Read SJ, Smith MI, Hunter AJ, Upton N, Parsons AA (2000) SB-220453, a potential novel antimigraine agent, inhibits nitric oxide release following induction of cortical spreading depression in the anaesthetized cat. Cephalalgia 20:92-99

71. MaassenVanDenBrink A, van den Broek RW, de Vries R, Upton N, Parsons AA, Saxena PR (2000) The potential anti-migraine compound SB220453 does not contract human isolated blood vessels or myocardium; a comparison with sumatriptan. Cephalalgia 20:538-545 